\title{
Method of Non Destructive Geophysical Mapping of the Environment
}

\author{
Olga Hachay¹, Oleg Khachay² \\ ${ }^{1}$ Institute of Geophysics, Ural Branch of Russian Academy of Sciences, Yekaterinburg, Russian Federation \\ ${ }^{2}$ Ural Federal University, Yekaterinburg, Russian Federation \\ Email: olgakhachay@yandex.ru, khachay@yandex.ru
}

How to cite this paper: Hachay, O. and Khachay, O. (2019) Method of Non Destructive Geophysical Mapping of the Environment. Open Journal of Geology, 9, 327-341.

https://doi.org/10.4236/ojg.2019.97022

Received: June 17, 2019

Accepted: July 16, 2019

Published: July 19, 2019

Copyright ( 2019 by author(s) and Scientific Research Publishing Inc. This work is licensed under the Creative Commons Attribution International License (CC BY 4.0).

http://creativecommons.org/licenses/by/4.0/ (c) (i) Open Access

\section{Abstract}

Problem statement: The method of non-destructive testing is needed in different aspects of geology, geomechanics and archeology, for instance: by testing the state of rock massifs in sault, kimberlites or rock shock mines, ancient archeological objects, or mapping for locating the wells drilling. The best method for solution these problems is based on using induction multi frequency electromagnetic field with use control sources on 3D planshet system of observation. Applied methods and design: Such method was developed at the Institute of Geophysics, Ural Branch of the Russian Academy of Sciences that allows its use to solve complex geological problems for mapping and monitoring the state of the environment. Its use allows the observation system to be used in such way that, on the one hand, it achieves flexible tunable detail of observations, and on the other hand, it organizes an input database that is close to the domain of definition of the inverse problem operator in the class of layered-block models with local hierarchical inclusions. This allows using regularization methods to obtain solutions that are in the class of equivalent, close to true. The specific possibilities of its use are determined by the technical side: the power of the excitation source, the sensitivity of the receiving system. Typical results: It had been developed a new method of mapping, processing and interpretation, realized in a set of programs that allow seeing the result of the searching structure of the environment in real time (that is after receiving some results during one day, we can see the results of the geoelectrical features of the structure and the state of that part of the environment). It had been suggested a three staged conception of interpretation that can be used not only for interpretation electromagnetic induction data and dynamic seismic data. Concluding note (Practical value/implications): In the considered case, this method contributed to the study of altered forms of kimberlitic bodies. The method underwent geological verification in various geological conditions where it had been used for 
mapping the geoelectrical structure. This allows us to offer a method for the development of innovative technology in the search for hydrocarbons in complex geological structures. The method was used in Egypt for research ancient archeological objects, located in Alexandria.

\section{Keywords}

Electromagnetic Planshet Method, Theory of Interpretation, Practice Use

\section{Introduction}

"Let me make some general remarks on the place of theoretical physics in relation to experimental physics. Theoretician as a foundation needs some general assumptions, the so-called principles, on the basis of which he can derive consequences" [1]. Among geophysical methods, a worthy place is occupied by electromagnetic studies with controlled sources. This is how a well-known geophysicist, theoretician and methodologist M.S. Zhdanov evaluates the role of electromagnetic research with controlled source [2] in solving the following problems of interest to geologists and geomechanics: determination of the depth and geometry (sometimes morphology) of the geo object under study; determination of the material-structural composition of the geo object; determination of the location of sub-vertical and sub-horizontal heterogeneities and, above all, macro-fracture zones; determination of filtration conditions for saturated porous objects; determination of the thermodynamic conditions for the occurrence of the researched object. Electrical methods were one of the first methods of geophysical prospecting, which were very widely used during the period from 1920 to 1930 for search of oil, gas, and ore minerals. The development of the practical use of electric fields for studying the structure of the Earth began with the pioneering work of the Schlumberger brothers. Over the years, electrical survey methods have undergone large changes, starting with a simple resistivity method and developing into a complex technology based on complex methods of electromagnetic research with natural and controlled sources. At that, not only methods and equipment changed, ideas about electromagnetic fields, their generation and measurements changed, and basic interpretative geoelectrical models changed: from one-dimensional to two-dimensional and three-dimensional models. The main thing was that non-destructive testing technologies were created that began to be used not only for mapping, but mainly for monitoring the state of the environment, without changing its structure, which is important for solving problems not only of geophysics, but also of ecology. A feature of the theory of electromagnetic methods was that its development proceeded simultaneously for artificial and natural sources of excitation. And here we can see the great contribution of our and foreign scientists (see bibliography in [2]) and [3]-[16]. In 1975 ([3] p. 29), on the initiative of Yu.P. Bulashevitch, electromagnetic sounding had been carried in the Ural, using an MHD generator. Such a 
sounding using a powerful pulsed magnetic dipole was made for the first time. The signal was recorded at a distance of up to $73 \mathrm{~km}$ and, despite the effects of surface heterogeneities, a variant of the section of the Urals crust and upper mantle was obtained by the electrical conductivity parameter. In the USSR, at that time, the MHD installations "Ural" and "Khibiny" were created, generating up to $50-100 \mathrm{MW}$ in a short time and up to $10-20 \mathrm{MW}$ in hundreds of hours. Research and development of MHD generators were widely deployed in the USSR, USA, Japan, Poland, India and other countries [17] [18] [19] [20]. Further progress in the development of geophysical methods of electrical exploration is associated with an increase in the depth of soundings; improved resolution of methods; increasing productivity and reducing the cost of electrical exploration; wide spread use of artificial (controlled) sources of primary fields. Such methods make it possible to obtain reproducible results at a convenient time for the researcher, putting the geophysical experiment into a number of active large-scale physical experiments [21].

In these paper it is developed new approach to process and interpret the data received for a new system of observation (planshet system), and in a new three staged concept of interpretation, that can be used not only for block layered model, but more complicated block layered model with hierarchical inclusions. That model is used now for testing the state of geological environment. More over that approach can be used for seismic dynamical system of observation.

\section{New Concepts of Interpretation of Electromagnetic Fields Data with Controlled Sources}

"Each individual geophysical survey method is associated with some uncertainty in the geological interpretation of the results of observations. This uncertainty decreases and the likelihood of correct interpretation increases with the application of several methods. Hence, naturally, there is a desire to use a complex of geophysical methods" [21]. What does "geological interpretation" of the results of geophysical observations mean? According to Yu.P. Bulashevitch [22], this means that in addition to the spatial distribution of the physical properties of the medium, determined in the framework of the impact on it by a particular field or registering a particular emission of the medium, it is important for us to determine the modern material composition and state of the geological environment. One of the variants of the modern reading of Yu.P. Bulashevitch's thought may be the following: the problem of integrating geophysical observations becomes more and more urgent due to the refinement of the interpretational model of a complex geological environment and the study of the mechanisms leading to its unsteady rearrangements [23] [24]. To study complex dynamic processes and their monitoring, a synchronous method of observing fields from various sources is already required. At the same time, unlike previously solved problems associated with geolocation of 3D layered-block media, when the unity of the complex interpretation model was achieved by superimposing individual models obtained by independent interpretation of data from various geophysical fields: 
gravitational, magnetic, seismic, electromagnetic, thermal, here it is necessary take into account the thermodynamic relationship of the material parameters of the medium. Hence, the interpretation process no longer represents an independent solution of inverse problems for each field separately. The operator of the inverse problem with complex interpretation is written out in the form of a system of equations for the fields connected by thermodynamic material relations. Examples include the algorithms for determining the mechanisms of convection in the Earth's mantle, the interpretation of natural-field anomalies associated with electro kinetic phenomena [25]. The theory of interpretation of geophysical fields is directly related to the solution of ill-posed problems and the theory of regularization had been created by the works of A.N. Tikhonov, V.K. Ivanov, M.M. Lavrentiev and their schools. As a result of its application, important results have been achieved in constructing models of local, regional and global distributions of physical properties in the Earth and other celestial bodies on which geophysical studies were carried out, within the framework of piecewise homogeneous models and a linear approximation to describe field propagation. The further development of geophysical methods in the direction of building more and more adequate models of environments and phenomena necessarily leads to more and more complex problems of mathematical geophysics, for which not only the inverse, but also direct problems become essentially incorrect [26] [27]. In this regard, on the one hand, it is necessary to develop a new concept of regularization for the simultaneous solution of a system of different types of operator equations. On the other hand, another approach is possible: the creation of equal-type interpretation algorithms based on solving equal-type operator equations that differ in the explicit form of the kernel of integral equations.

Using the second approach and the ideas of A.V. Tsirulskiy about a two-stage interpretation of potential stationary fields, we created a unique concept of three-stage interpretation electromagnetic and seismic fields [27]. In this concept, the idea of filtering the input data into the domain of definition of the solution of a one-dimensional inverse problem operator and the idea of approximating anomalous fields with a class of fields with predetermined properties [28] is used for regularization. Algorithms that implement all the stages of interpretation have been developed, allowing investigating the applicability of this approach to the analysis of practical data [27] [28]. For the simultaneous use of data from various fields, the basic principles of the construction of a real observation systems were developed, allowing, on the one hand, to obtain a database consistent in the normal field, on the other hand, to realize $3 \mathrm{D}$ interpretation in the framework of unified algorithms. Examples of practical application indicate the high information content of the proposed integration [29] [30].

Consider the component parts of this concept:

1) At the first stage, the electromagnetic and seismic parameters of the horizontally layered-block medium are determined, containing elastic and geoelectrical heterogeneities.

2) At the second stage, the anomalous field is selected by the field of a system 
immersed in the medium (with the physical parameters determined at the first stage) of singular sources that are equivalent in terms of the field to local geoelectrical and elastic heterogeneities. At the same time, a geometric model of individual local heterogeneities or groups and their mutual arrangement inside a layered-block containing medium are determined.

3) At the third stage, the surfaces of the desired heterogeneities are determined depending on the values of the physical parameters of the anomalous objects.

\section{Practical Implementation of the New Concept}

The following theoretical and practical results were obtained in the paper [28]:

1) A unified observation system for alternating electromagnetic and seismic fields using a local excitation source has been developed and tested. The choice of the type of excitation source was determined by the following: a) a uniform geometry of the normal field, b) the absence of one or several components in the measured field in the case of a quasi-layered medium. Such properties in the case of the electromagnetic field have a vertical magnetic dipole, in the case of a seismic field-a vertically acting force. The presence of a local source of excitation allows regularly for a given network of observations to realize overlaps at different angles of visibility (the source of excitation towards the heterogeneity). Three components of the magnetic field and three components of the field of elastic displacements as functions of spatial coordinates and time are used as input data for interpretation. For ground-based observations, the data are recorded on the day surface for a set of distances between the source and receiver as a function of time. Analysis of the solution of direct problems for seismic and electromagnetic cases, showed [29] [30] that a single computational approach can be realized when interpreted after a preliminary transfer of electromagnetic data to the real axis, and seismic to an imaginary axis of the complex frequency plane. In this case, the whole further process of interpretation must be conducted on this plane, without going back to the time domain.

2) For the implementation of the second stage, namely the analysis of the anomalous field, the ratios of the modules of the horizontal components of the seismic and magnetic fields were introduced as a function of the spatial coordinates and the real or imaginary frequency parameter. In the cylindrical coordinate system, these are the $\varphi$-components to $\rho$-components, which have the meaning of seismic and geoelectrical heterogeneity parameters and quantitatively characterize the degree of medium deviation from the horizontally layered one $(\delta \%)$. These parameters are selected using the approximation construction [31] [32] [33], built on the basis of explicit expressions for the fields of singular sources of the following form: for a seismic field, this is a set of point sources of forces acting on a segment of finite length in an arbitrary direction, for an electromagnetic field-current lines of finite length. Analysis of the direct problem for a seismic case under the action of a source located in the horizontal plane allows us to conclude that to achieve unity of morphology of the selected anomal- 
ous fields, the system of singular sources in the form of closed current contours-horizontal magnetic dipoles-is preferable for the electromagnetic field. Despite the fact that both systems are complete and suitable for the selection of anomalous electromagnetic fields, with joint integrated interpretation of seismic and electromagnetic fields, in addition to changing the design type, it makes sense to introduce an additional parameter in the electromagnetic case, which is the ratio of electrical horizontal components $\rho$ and $\varphi$, the spatial distribution of which is more closely related to the distribution of the seismic heterogeneity parameter in its shear part and therefore in electromagnetic case it is necessary to measure further components of the horizontal electric or magnetic field corresponding derivatives. Algorithms that implement all the stages of interpretation have been developed, allowing investigating the applicability of this approach to the analysis of practical data [29] [30]. For the simultaneous use of data from different fields, the basic principles of the construction of a real observation systems were developed, allowing, on the one hand, to obtain a database harmonized across the normal field, and on the other hand, to realize 3D interpretation in the framework of unified algorithms. Examples of practical application indicate the high information content of the proposed integration [31] [32] [33]. The implementation of the described methodology is carried out using the A.I. Chelovechkov equipment developed for shallow induction electromagnetic research, as well as Sinus seismic equipment developed by L.N. Senin at the Institute of Geophysics, Ural Branch of the Russian Academy of Sciences. Thus, the Institute of Geophysics has developed a 3-D electromagnetic planshet frequency-geometric, instrumental-methodical and interpretational complex based on a controlled excitation source in the form of a vertical magnetic dipole, implemented as an instrument complex, a planshet observation system, programs for processing, interpretation and visualization in view 3-D models of the data. The equipment was created by Ph.D. A.I. Chelovechkov, methodology, theory of interpretation-Dr. Sc. O.A. Hachay, software implementation-Ph.D. Khachay A.Yu., Ph.D. Khachay O.Yu.

\section{Practical Use of a Planshet Technique Using Induction Electromagnetic Technique}

Within the framework of the project approved by the Ministry of Natural Resources of the Russian Federation, together with the Ural Committee of Natural Resources, the described complex methodological interpretation was effectively used to carry out prospecting to clarify the structure of the platinum placer on the flanks of the Lobva deposit. Predicted geophysical sections were confirmed by drilling data and opening work. The planshet electromagnetic technique was used to solve the problem of tracing serpentinites in the Urals (grant RFBR No. 99-05 64586). The complex seismic and electromagnetic methods, adapted to study the structure and state of rock areas, are used in the underground version as a volume observation system, first implemented in mine conditions [34] (RFBR grant No. 99-05-64371). The experience of the development of hydro- 
carbon deposits in Western Siberia quite definitely testifies of a significant localization of oil and gas in the structure of deposits. The transverse dimensions of local deposits of high flow wells often do not exceed several hundred meters and are separated by hydro dynamically impermeable rocks that retain the primary structure of clay sediments [35]. On the Yamal semi island, in the regional plan, a linearly elongated strip of sub-vertical zones of destruction is distinguished, extending from the north-north-west to south-southeast of the Nurminsky shaft axis and controlling all major hydrocarbon fields in this area. After crossing the southern part of the Ob Bay, its continuation in Western Siberia is clearly visible ([35] see bibliography).

The high degree of localization of increased productivity sites associated with sub-vertical zones of destruction can be judged by the example of the Yem-Egovsky field (Western Siberia): on a plot of 1 sq. million $\mathrm{km}$ one of about 100 wells gave 95\% of all oil produced. Small transverse dimensions of the reservoir area, high production rate and total cumulative production completely determine the field-geological model of this field and its analogues in a new way. Such a model is rather similar to the model of a vein ore or diamond ferrous deposit [35].

\section{Illustration of the Implementation of the Developed Methodology in the Diamond-Bearing Province}

Let us give an example of its use in the Krasnovishersk region of the Perm domain.

\subsection{Geological Description of Krasnovishersk Perm Domain}

In Krasnovishersk region (western slope of the Urals), two types of diamond deposits are exploited: quaternary alluvial placers located in the Bolshoi Shchugor, Bolshoi and Severniy Kolchim river beds and ancient buried placers confined to elevated areas of the relief, which were previously considered deposits in erosion depression [36]. After the discovery of the deposits, the Ishkovskiy career and the Northern Rassolnaya geological positions of deposits of this type became more definite. The Ishkovskiy open-pit (career) mine is located on the water parting of the Volynka and Efimovka rivers-inflows of the Bolshoi Shchugor river. Information about the geological structure of the field will be given according to the results of [36] [37]. The deposit is located on the contact of carbonates of the Kolchim suite and the sandstones of the Takata suite, having a meridional trend and gentle incidence angles. Kolchim formation carbonates are represented by dolomite and recrystallized lime stones with no visible stratification. They are widely manifested by karsts phenomena. Karst develops most intensively at the contact of lime stones with sandstones of the Takata formation, which extends to the day surface. As a result, the contact acquires a fancy curved configuration complicated by karst craters and sandstone blocks that have fallen into them. In some cases, karst craters develop to the full thickness of limestone, 
and in these craters are blocks of Takata sandstones that are far from contact, surrounded by carbonate rocks. The rocks of the Takata formation are represented on the deposit by gravelites, coarse-grained sandstones and interlayers of green-white unlithified clays with a thickness of $1.5-2.0 \mathrm{~m}$, layered down with quartz sandstones with a thickness of $0.1-0.15 \mathrm{~m}$. At the base of the section, lenses of rarely-pebble conglomerates with sizes up to several meters and with a thickness of up to $0.2 \mathrm{~m}$. The origin of such lenses is connected, apparently, with the destruction of more ancient conglomerates and canning of debris formed during weathering in quartz sandstone and in gravelites. The productive horizon is disintegrated quartz sandstone and gravelites of the Takata suite, which lie on the karsted surface of the limestone of the Kolchim suite, separated from the massive limestone by a layer of karst clay. Separate diamond crystals had been found in massive sandstones. The disintegration of sandstones occurs not only where the contact of carbonates and sandstones reaches the surface, but also at depth, and massive sandstones, as a rule, lie on the disintegrated ones.

The presence of karst and karst carbonate clays under Takata quartz sandstones allows us to conclude that the weathering crust, which is fixed at depth, along the contact surface of Takata and Kolchim deposits, is Paleozoic, formed before the formation of sandstones and Takata gravelites, which within the Kolchim anticline also represent continental formations. The same conclusion was reached by Y. Becker and co-authors based on a study of the lithology of the deposits of the Takata suite and the geological structure of the diamond ferrous placer at the Ishkovskiy open-pit (career) mine [37] [38]. The Mesozoic and Cenozoic weathering processes are superimposed on the Paleozoic weathering crust, especially strongly developed at the contact of carbonate rocks with sandstones that extend to the surface. The occurrence of the productive horizon in the Paleozoic weathering crust and its overlapping with deposits of the Takata formation allows us to consider the Ishkovskiy open-pit mine as an ancient Paleozoic alluvial deposit [39].

The above information from the geology of the formation of the Ural diamond ferrous placers shows that the most important requirement for the instrumental and methodical geophysical complex and the method of interpreting geophysical data for studying the structure of the placer and their sources is the fundamentally new resolution of methods in the absence of a priori information about the site under study. This was achieved in the framework of the planshet electromagnetic frequency geometrical research technique developed at the Institute of Geophysics, Ural Branch of the Russian Academy of Sciences, which is used for mapping and monitoring of complex geological media in the surface and underground (mine) version [31] [32] [40]. Adaptation of this technique to underground research in mine workings to determine the structure of rock massifs, the state and their dynamics under anthropogenic impact made it possible to conduct volume geophysical studies in the geological environment and to identify 
the main structural factors and criteria of the state of the geological environment. The technique used refers to the geophysical methods of non-destructive testing. It differs from previously known methods of $\mathrm{x}$-ray or tomography observation systems and the subsequent method of interpretation. These results made it possible to establish an important morphological factor: diamond ferrous areas in the form of placer deposits gravitate to high-resistance structures, which are either the sources themselves or are structures that block the spreading and further movement of diamonds. These structures are mapped to depths of $70-100$ $\mathrm{m}$ and deeper. The use of methods commonly used for in-depth studies does not allow them to be distinguished with sufficient resolution. It turns out a fairly uniform resistance section, which is a consequence of the integration of high and low-resistance structures. The resulting geoelectrical cuts in kimberlites strata are high-resistance with a resistance comparable to the resistance of high-resistance structures controlling the developed placers. In addition, the applied technology allowed identifying new depressions, thereby expanding the area of possible diamond mining. One of them is confirmed by detailed geological borehole verification. It is located on the Northern Rassolnaya placer [40]. The obtained data of geological-geophysical structure and diamond-bearing nature of the rocks of the Kolchimsky anticline suggest that the primary sources of diamonds are found in pre-Silurian sediments, and their age, taking into account the fact that the Pomenennovskaya and Poldovskaya formations fall out of the Paleozoic section, is not less than 670 million years. With the destruction of primary sources, diamonds were initially concentrated in terrigenous sediments of the Kolchim Formation, and during erosion of these sediments they fell into the base of the Takata formation. It is possible that the ancient diamond ferrous placers can be found in terrigenous sediments of the Kocheshor suite or in the upper part of the section of the Ilyavozh suite. If this assumption is true, then indigenous sources should be sought in the rocks of the Kocheshor, Ilyavozh and more ancient suites, but this causes another problem that complicates the search for indigenous sources. Rocks of Kocheshor and more ancient suites underwent deformation and their primary horizontal occurrence changed to sub vertical. According to E.V. Shipilova, the deformation is associated with the Baikal orogenesis, manifested within the Timan-Pechersk Plate. The bodies of kimberlites must undergo a corresponding transformation if they were introduced before the folding stage. Naturally, after such a transformation, traditional methods of searching for kimberlites in the area of Krasnovishersk diamond deposits are not suitable.

\subsection{Geophysical Results Using the Planshet Methodology}

Detailed geophysical methods focused on models of complex-structured environments, one of which was developed at the Institute of Geophysics of the Ural Branch of the Russian Academy of Sciences, will play an important role here and in the testing process showed sufficient resolution for comparison with the geological data of drilling (Figures 1-3). 
In Figure 1 we can see the whole profiles that had been developed using the method of wide profiles that are presented as a part of a planshet system. We see also the place Ishkov career, where had been developed and used the area planshet system. The same system had been developed at the placer South Rassolnaya, for what the geoelectrical sections agreed with the drilling data. That result allows us to develop the whole work for mapping the areas of diamond deposits for instance in the Ishkov career.

In Figure 2 we can see a fragment of the geoelectrical section of the profile 0 1. We see that the structure is very blocked and the resistivity of them differs from $20-5000 \mathrm{ohm} \cdot \mathrm{m}$. We can see the part of the profile (pickets 26-28) is very conductive. Really from Figure 3 we see that for these pickets we observed very high parameter of heterogeneity that corresponds with the geological fault.

That work was performed jointly with the Institute of Mineralogy, Ural Branch of the Russian Academy of Sciences under the guidance of Corresponding Member of the Russian Academy of Sciences V.N. Anfilogov with the support of the Russian Foundation for Basic Research, project 04-05-64136.

In addition, we carried out parametric electromagnetic studies in the framework of the same technology in the kimberlites mines International and Aikhal in Eastern Siberia.

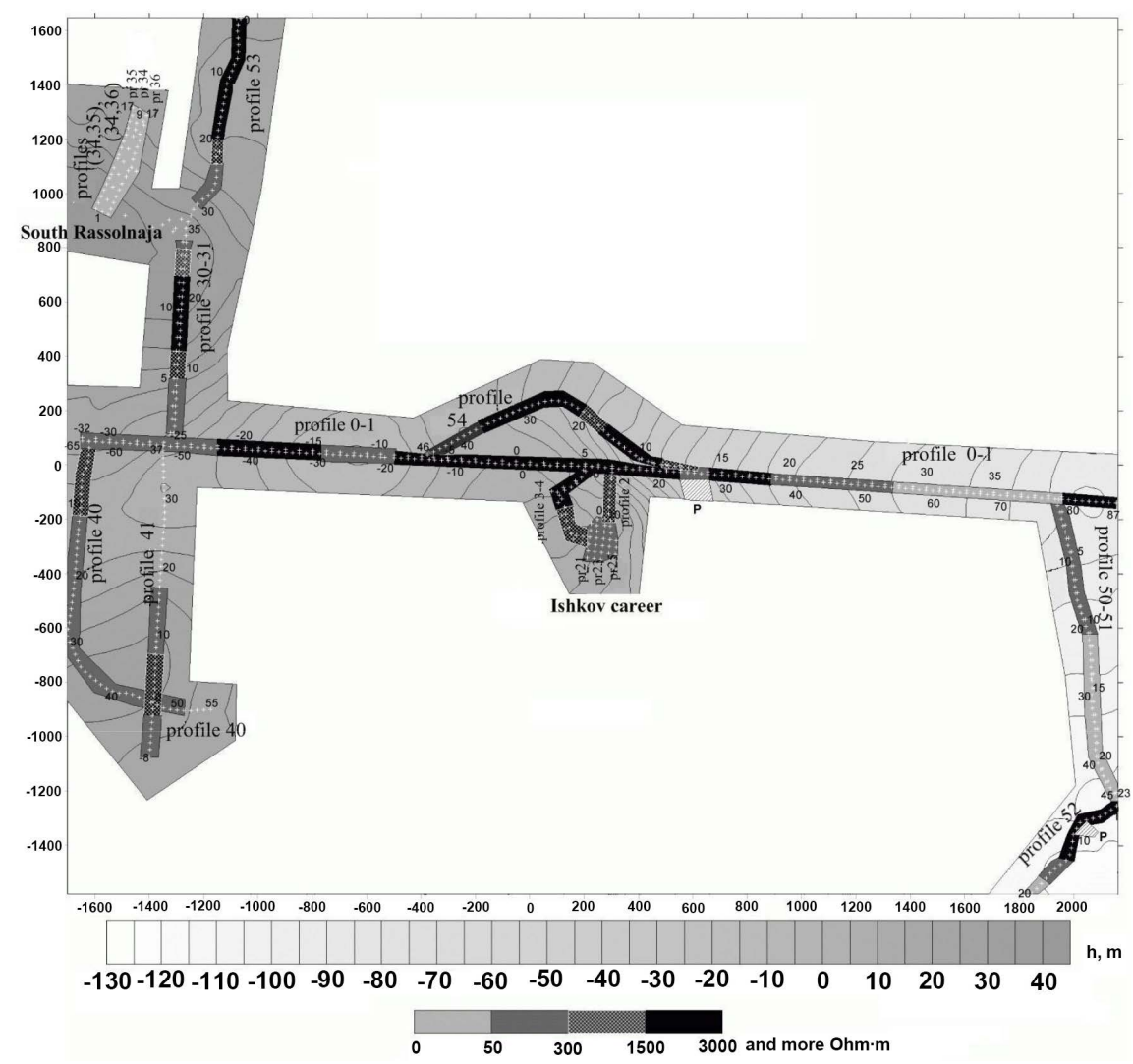

Figure 1. Scheme of electromagnetic research on the Krasnovishersk diamond area using the planshet method. First legend is the relief of the area, the second-the resistivity of the block massive on the profiles. 


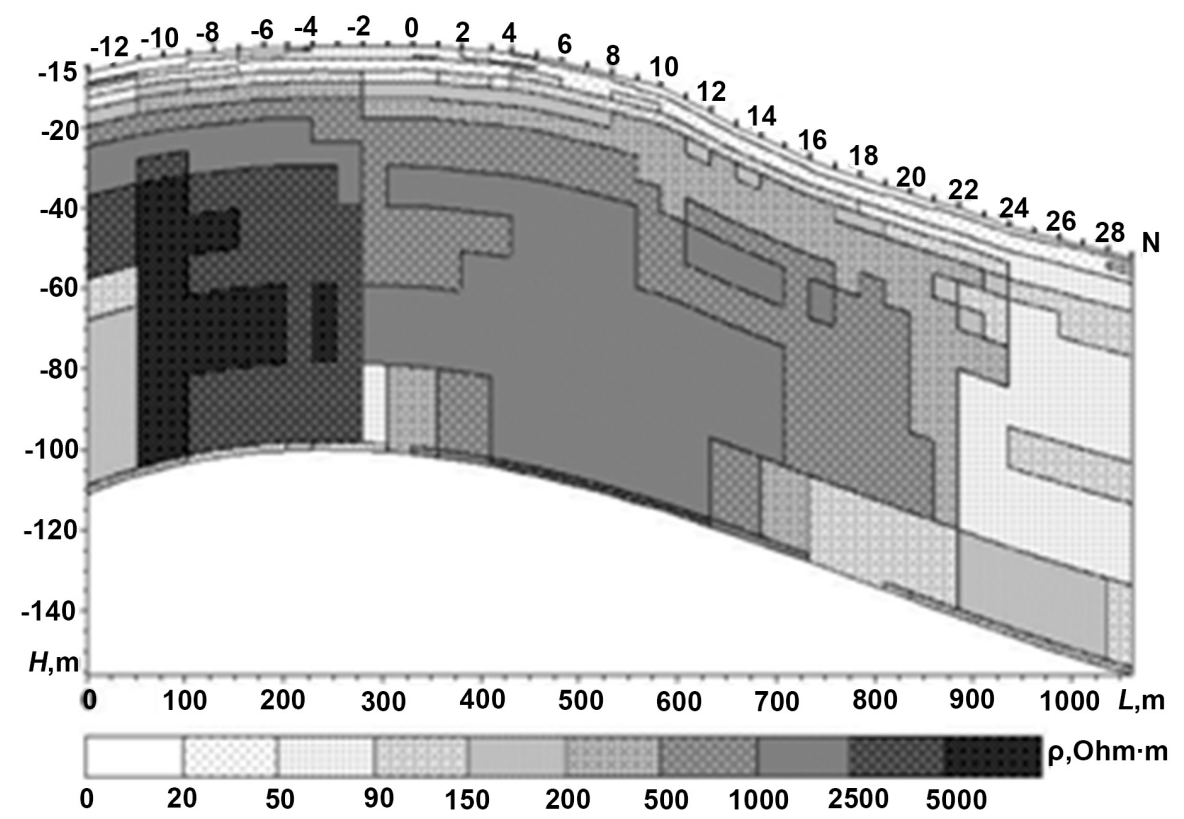

Figure 2. The resistivity section along the part of the profile 1 (Figure 1).

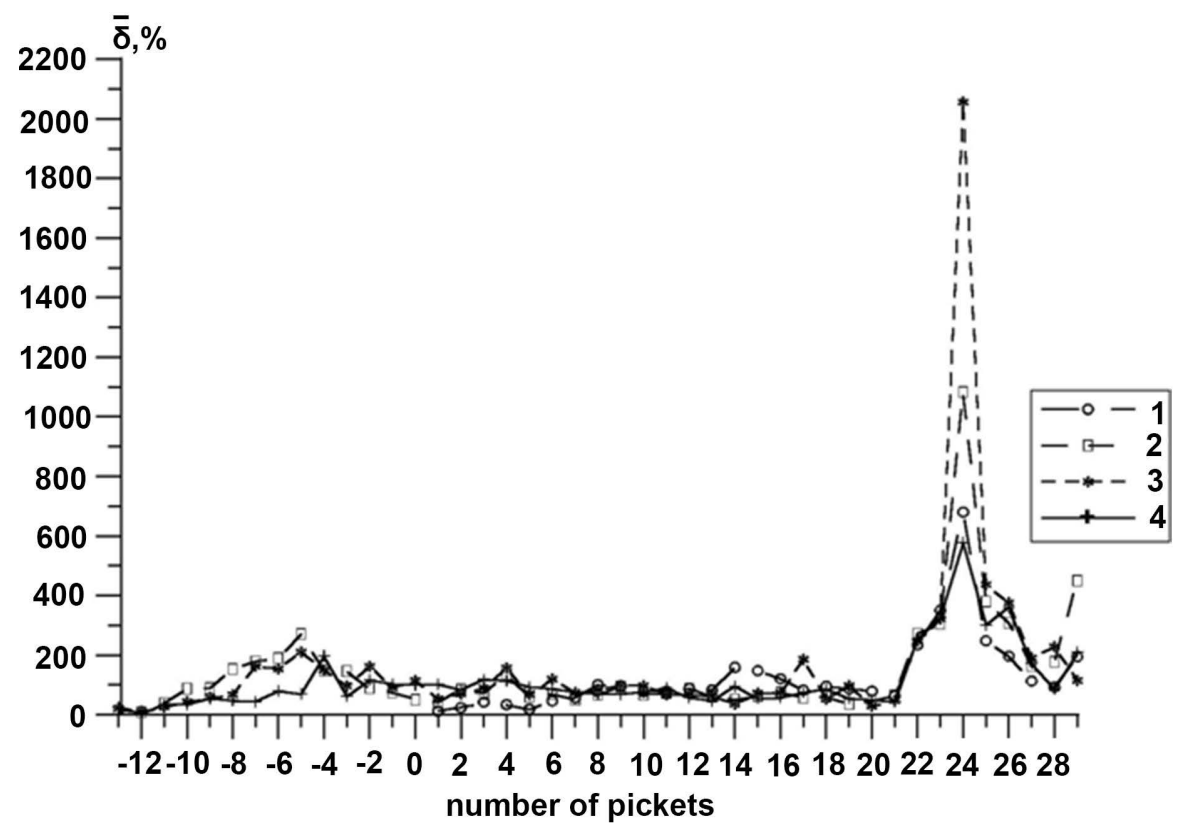

Figure 3. The distribution of the parameter heterogeneity along the part of profile 1 (Figure 1). Legend: $1-20.3 \mathrm{kHz}, 2-5.08 \mathrm{kHz}, 3-1.27 \mathrm{kHz}, 4-635 \mathrm{~Hz}$.

The used method allows its adjustment to solve complex geological problems. Its application allows the observation system to be implemented in such a way that, on the one hand, it achieves flexible tunable detail of observations, and on the other hand, it organizes an input database that is close to the domain of the inverse problem operator in the class of layered-block models with local inclusions. This allows using the regularization methods to obtain solutions that are in the class of equivalent, close to true. The specific possibilities of its imple- 
mentation are determined by the technical side: the power of the excitation source, the sensitivity of the receiving system. In the considered case, this method contributed to the study of modified forms of kimberlites bodies, which arose during the evolution of the diamond-bearing province. The method underwent geological verification in various geological conditions. This allows us to offer a method for the development of innovative technology in the search for hydrocarbons in the complex geological structures of Yamal and adjacent territories.

\section{Conclusion}

The new approach, which forms the basis of an integrated seismic and electromagnetic research method, has shown its viability and practical significance in the study of rock massifs of various material compositions. Seismic and electromagnetic information is mutually complementary in the mapping and prediction of the behavior of an array that is under technogenic influence. It relies on the use of active methods of geophysical monitoring using controlled concentrated sources of excitation, allowing spatial movement along a given observation system with overlapings. The complex technique has a high resolution that does not concede to tomographic studies, and the created software allows you to restore the volume geoelectric and seismic model of the array, which is used to calculate the volume distribution of the stress tensor in the array [23] [41] [42]. The implementation of the technique can, with a decrease in the excited and measured frequencies of an alternating magnetic field with a given detail, either directly map the structure of the hydrocarbon deposit, or use the detailed seismic information on these zones for setting industrial wells with a sharp reduction in additional preliminary drilling. Currently, such equipment is available in the IGF UB RAS. The developed planshet induction technique using a controlled source of excitation was included in the complex of electromagnetic methods used in the Arab Republic of Egypt for solving problems of shallow geophysics and archeology [43] [44].

\section{Conflicts of Interest}

The authors declare no conflicts of interest regarding the publication of this paper.

\section{References}

[1] Einstein, A. (1967) V1. Science, Moscow.

[2] Zhdanov, M.S. (2010) Electromagnetic Geophysics: Notes from the Past and the Road Ahead. Geophysics, 75, 75A49-75A66. https://doi.org/10.1190/1.3483901

[3] Bulashevitch, Y.P. (2014) The Founder of academic Geophysics in the Urals. RIO UB RAS, Ekaterinburg.

[4] Bursian, V.R. (1972) The Theory of Electromagnetic Fields Used in Electrical Prospecting. Nedra, Leningrad.

[5] Vanyan, L.L. (1965) Fundamentals of Electromagnetic Soundings. Nedra, Moscow. 
[6] Velikin, A.B. and Frantov, G.S. (1962) Electromagnetic Fields Used in Inductive Methods of Electrical Prospecting. Review of Foreign Literature. Gostoptekhizdat, Leningrad.

[7] Kormiltsev, V.V. and Mezentsev, A.N. (1989) Electro Prospecting in Polarizing Media. Ural Branch of the Academy of Sciences of the USSR, Sverdlovsk.

[8] Svetov, B.S. (1973) Theory, Methods of Interpretation of Materials of Low-Frequency Inductive Electrical Prospecting. Nedra, Moscow.

[9] Sidorov, V.A. (1985) Impulse Inductive Electrical Prospecting. Nedra, Moscow.

[10] Tabarovsky, L.A. (1975) Application of the Method of Integral Equations in Problems of Geoelectrics. Nauka, Novosibirsk.

[11] Aksenov, V.V. (2002) Electromagnetic Field of the Earth. SB RAS, Novosibirsk.

[12] Nazarny, S.A. and Komarov, V.A. (2001) Caused Seism Electric Polarization. VIRG, S.P. University, Saint Petersburg.

[13] Svetov, B.S. (2007) Foundations of Geoelectrics. TsGEMI RAN, Moscow.

[14] Berdichevsky, M.N. and Dmitriev, V.I. (2009) Models and Methods of Magnetotellurics. Scientific World, Springer, Berlin, |Heidelberg. https://doi.org/10.1007/978-3-540-77814-1

[15] Kaufman, A.A. (2000) Introduction to the Theory of Geophysical Methods. Electromagnetic Fields. Nedra. Moscow.

[16] Astrakhantsev, G.V. (1988) Induction Sensing in the Study of Contrasting in Electrical Conductivity Environments. UB RAS, Sverdlovsk.

[17] Astrakhantsev, G.V., Babakov, Y.P., Bulashevitch, Y.P., et al. (1977) Induction Sounding of the Earth's Crust in the Urals Using MHD Generator. DAN USSR, 237, 808-812.

[18] Astrakhantsev, G.V., Dyakonov, B.P., Bulashevitch, Y.P., et al. (1979) The Study of the Electrical Conductivity of the Earth's Crust in the Urals. Izvestiya USSR Academy of Sciences. Physics of the Earth, 1, 15-26.

[19] Velikhov, E.P. and Volkov, Y.M. (1982) Prospects for the Development of Pulsed MHD Energy and Its Application in Geology and Geophysics. In Deep Electromagnetic Sounding Using Pulsed MHD Generators, Academy of Sciences of the USSR. Apatity, 5-25.

[20] Gorbunov, G.I., Belkov, I.V., Pavlovsky, V.I., Zhamaletdinov, A.A., et al. (1979) Deep Electromagnetic Sounding with an MHD Generator on the Kola Peninsula. DAN USSR, 247, 578-582.

[21] Hachay, O.A. (2002) Ideas Yu.P. Bulashevich in Modern Understanding of the Problem of Integration of Geophysical Methods. Ural Geophysical Bulletin, IGF, Ural Branch of the Russian Academy of Sciences, 3, 42-46.

[22] Bulashevitch, Y.P., Dyakonov, B.P., et al. (1980) Methods and Results of Building a Complex Model of the Earth's Crust and Upper Mantle of the Urals. In: Tektonosphere of Ukraine and Other Regions of the USSR, Kiev, 181-195.

[23] Hachay, O.A. (2000) Complex Geophysical Studies (Theory and Practical Results) Ural Geophysical Bulletin IGF UB RAS, 1, 107-110.

[24] Hachay, O.A. and Khachai, Y.V. (1993) On the Identification of the Physical Mechanisms of Mantle Convection Based on the Inverse Problem Method. Geology and Geophysics, 6, 15-21.

[25] Kormiltsev, V.V. and Hachay, O.A. (1995) Mechanic- and Seism Electric Phenomena in Rocks. In: Non-Classical Geoelectric, OIFZ RAS, Saratov, 22-29. 
[26] Samarsky, A.A. and Kurdyumov, S.P. (1989) Paradoxes of the Multivariant Non-Linear World-The World around Us. Hypotheses and Forecasts M.: Knowledge, 8-29.

[27] Hachay, O.A. (1996) Three-Stage Method of Interpretation of Variable Electromagnetic Fields and Its Practical Implementation. In: Electromagnetic Research with Controlled Sources. RAS, St. Petersburg, 30-31.

[28] Hachay, O.A. and Novgorodova, E.N. (1997) The Experience of Areal Induction Studies of Highly Inhomogeneous Geoelectric Media. Physics of the Earth, 5, 60-64.

[29] Hachay, O.A. (1980) Unified Method for Solving the Inverse Problem of Electromagnetic Soundings for a One-Dimensional Medium. Izvestiya USSR Academy of Sciences. Physics of the Earth, 5, 51-60.

[30] Hachay, O.A. (1991) On the Solution of the Inverse Problem for Three-Dimensional Variable Electromagnetic Fields. Izvestiya Academy of Sciences USSR. Physics of the Earth, 6, 50-57.

[31] Hachay, O.A., Novgorodova, E.N. and Bodin, V.V. (1999) On the Problems of Shallow Geoelectrics and Some Results of Their Solution. Physics of the Earth, 5, 47-53.

[32] Hachay, O.A and Druzhinin, V.S. (2000) Integrating Three-Dimensional Methods of Seismic and Electromagnetic Research to Solve Monitoring Problems. Exploration and Protection of Mineral Resources, 2, 22-24.

[33] Hachay, O.A., Novgorodova, E.N. and Khachai, A.Y. (2000) Mapping of ThreeDimensional Conducting Zones Using Areal Observation Systems in the Framework of the 3-D Frequency-Geometric Technique. Geology and Geophysics, 41, 1331-1340.

[34] Hachay, O.A., Novgorodova, E.N. and Khachai, A.Y. (2003) Investigation of the Resolution of the Planshet Electromagnetic Method for Active Mapping and Monitoring of Heterogeneous Geoelectric Media. Physics of the Earth, 1, 27-37.

[35] Megerya, V.M. (2009) Search and Exploration of Hydrocarbon Deposits Controlled by Geosoliton Degassing of the Earth. Locus Standy, Moscow.

[36] Anfilogov, V.N., Korablev, A.G. and Kabanova, L.Y. (2001) Features of the Geological Structure and Genesis of Krasnovishersk Diamond Deposits. In: Diamonds and Diamond Content of the Timan-Pechersk Region, Geoprint, Syktyvkar, 69-71.

[37] Becker, Y.R., Bekasova, N.B. and Ishkov, A.D. (1970) Diamond Ferrous Placers in the Devonian Sediments of the Northern Ural. Lithology and Minerals, 4, 65-75.

[38] Sokolov, B.N. (1982) Formation of Placers of Diamonds. Science, Moscow.

[39] Shuysky, V.P. (2000) Paleontological characteristics of the Takata Formation of the Krasnovishersk District (Northern Ural). In Yearbook 1999: Information Collection by the Institute of Geology and Geochemistry, Yekaterinburg, 31-35.

[40] Hachay, O.A., Khachai, O.Y. and Kononov, A.V. (2008) The Use of New Geophysical Technology in the Study of the Relationship of the Primary Source and Placer of Diamonds. Exploration and Protection of Mineral Resources, 2, 58-60.

[41] Hachay, O.A. (1997) The Three-Stage Concept of Common Interpretation for 3-D Electromagnetic and Seismic Fields and Some Results of Its Practical Realization. Engineering and Environmental Geophysics for the 21 st Century, China, Chengdu, 286-292.

[42] Hachay, O.A., Bodin, V.V., Novgorodova, E.N., et al. (1997) A New Complex Near-Surface Electromagnetic and Seismic Technique for 3-D Research of Heterogeneous and Unstationary Medium. 181-189. 
[43] Atya, M.A., Hachay, O.A., Abdel Latif, A., et al. (2010) Geophysical Contribution to Evaluate the Hydrothermal Potentiality in Egypt: Case Study: Hammam Pharaoh and Abu Swiera, Sinai, Egypt. Earth Sciences Research Journal, 14, 44-62.

[44] Atya, M.A., Khachay, O.A., Soliman, M.M., et al. (2010) CSEM Imaging of the Near Surface Dynamics and Its Impact for Foundation Stability at Quarter 27,15 ${ }^{\text {th }}$ of May City, Helwan, Egypt. Earth Sciences Research Journal, 14, 76-87. 